\title{
Spontaneous regression of transverse colon cancer with high-frequency microsatellite instability: a case report and literature review
}

Nozomi Karakuchi ${ }^{1}$, Manabu Shimomura ${ }^{1 *}$, Kazuhiro Toyota ${ }^{1}$, Takao Hinoi $^{2}$, Hideki Yamamoto ${ }^{3}$, Seiji Sadamoto ${ }^{1}$, Koichi Mandai ${ }^{4}$, Hiroyuki Egi ${ }^{5}$, Hideki Ohdan ${ }^{5}$ and Tadateru Takahashi ${ }^{1,5}$

\begin{abstract}
Background: Spontaneous regression (SR) of colorectal cancer (CRC) is extremely rare, and only few cases have been reported. Although it is not yet clarified, a plausible mechanism for SR of CRC is an immunological event.

Case presentation: In this report, we present the case of SR of primary CRC in a 78-year-old man. Preoperative colonoscopy was performed, and a type 2 tumor measuring $30 \mathrm{~mm}$ in diameter in the transverse colon was detected. The biopsy revealed a poorly differentiated adenocarcinoma. Colectomy was performed 2 months after initial colonoscopy. During the surgery, only a 10- $\mathrm{mm}$ ulcer harboring a polypoid lesion measuring $8.5 \mathrm{~mm}$ was detected in the resected tissue; no other masses or carcinoma cells were seen on histological examination. Afterwards, the biopsy specimens were reanalyzed, and immunohistological analysis verified this as adenocarcinoma with stroma-infiltrating lymphocytes. Further analysis revealed a loss of two mismatch repair proteins, suggesting sporadic high-frequency microsatellite instability (MSI-H).

Conclusion: According to previous literature, a common site of SR in CRC is the proximal colon, which is a feature of MSI-H CRC. However, our report showed a rare case of SR of CRC, which was in the transverse colon, with MSI-H present. This report indicates a relationship between immunological features of MSI-H and the occurrence of SR of CRC. A better understanding of this phenomenon and the mechanisms involved will have significant preventive and therapeutic implications for CRC, including anti-PD-1 immune checkpoint inhibitor therapy.
\end{abstract}

Keywords: Colorectal cancer, Spontaneous regression, Microsatellite instability

\section{Background}

Spontaneous regression (SR) of malignant tumor is defined as their partial or complete disappearance in the absence of all treatment or in the presence of treatment that is considered inadequate to exert a significant influence on neoplastic disease [1,2]. Although the detailed mechanism of SR has not been fully understood yet, an immunological event is reported as one of the possible causes of SR.

SR of colorectal cancer (CRC) is known to be extremely rare, accounting for less than $2 \%$ of all the

\footnotetext{
* Correspondence: manabus761215@gmail.com

${ }^{1}$ Department of Surgery, National Hospital Organization Higashihiroshima Medical Center, 513, Jike, Saijyo-cho, Higashihiroshima, Hiroshima 739-0041, Japan

Full list of author information is available at the end of the article
}

SR cases [3]. Herein, we report a rare case of SR of transverse colon cancer in a 78-year-old man. We conducted immunostaining and found that the expression levels of the mismatch repair proteins (MMRs) were decreased, indicating that this tumor was a CRC with high-frequency microsatellite instability (MSI-H). Recent studies have reported the effectiveness of an anti-programmed cell death 1 (PD-1) antibody treatment for MSI-H CRC, because of its immunological characteristics [4]. In this paper, we summarized all the similar reported cases and reviewed the possible interactions between $\mathrm{SR}$ and MSI-H CRC.

(C) The Author(s). 2019 Open Access This article is distributed under the terms of the Creative Commons Attribution 4.0 International License (http://creativecommons.org/licenses/by/4.0/), which permits unrestricted use, distribution, and reproduction in any medium, provided you give appropriate credit to the original author(s) and the source, provide a link to the Creative Commons license, and indicate if changes were made. The Creative Commons Public Domain Dedication waiver (http://creativecommons.org/publicdomain/zero/1.0/) applies to the data made available in this article, unless otherwise stated. 


\section{Case presentation}

The patient was a 78-year-old man who had consulted the physician for paroxysmal atrial fibrillation (pAf), chronic heart failure, and chronic renal failure. Anti-coagulant therapy was administered to the patient for pAf. At a follow-up examination, the patient complained of tarry stool. The patient had no family history of cancer.

A colonoscopy was performed and revealed a type 2 tumor in the transverse colon measuring $30 \times 30 \mathrm{~mm}$ (Fig. 1a). Marking was performed by injecting a black dye into the submucosal layer, near the tumor, for future surgical resection (Fig. 1b). Biopsy specimens from the tumor suggested a poorly differentiated adenocarcinoma (Fig. 3a, b). Moreover, laboratory examinations revealed no remarkable abnormality: the carcinoembryonic antigen (CEA) and carbohydrate antigen 19-9 (CA19-9) levels were $3.1 \mathrm{ng} / \mathrm{ml}(<5.0)$ and $3.4 \mathrm{U} / \mathrm{ml}(<37)$, respectively. A computed tomography $(\mathrm{CT})$ scan revealed wall thickening, which was the basis for diagnosing the lesion, as the tumor invaded the muscularis propria (T2); moreover, there was no evidence of lung, liver, or lymph node metastases. The clinical diagnosis was T2N0M0, stage I according to the TNM classification (UICC 8th edition).

Laparoscopy-assisted colectomy was carried out 2 months after the initial colonoscopy. The patient did not receive any alternative medications, such as supplements, vitamins, and immunotherapy. We resected the colon, including the marking made during colonoscopy. The resected specimen revealed a $10-\mathrm{mm}$ ulcer with a polypoid lesion of $8.5 \mathrm{~mm}$ in the center (Fig. $2 \mathrm{a}$ ), but there was no type 2 tumor. The formalin-fixed specimen was cut into $3-5 \mathrm{~mm}$ slices. Histological examination demonstrated a marked nonspecific granulation of tissue, indicating fibrillization under the mucous membrane and sloughing off of the epithelium (Fig. 2b). Moreover, no cancer cells were found in the scar tissue (Fig. 2c, d). The dissected lymph nodes also did not show the presence of cancer cells. We used immunohistological staining to further evaluate the biopsy specimen. The findings showed that the tumor cells were strongly positive for AE1/AE3 (Fig. 3c) and positive for p53 (Fig. 3d), indicating that it was an adenocarcinoma. These findings suggested SR of colon cancer. Hematoxylin-eosin staining showed poorly differentiated adenocarcinoma, with tumor-infiltrating lymphocytes (TILs) in the tumor stroma. Based on these pathological features including poorly differentiated adenocarcinoma and TILs and the tumor location in the proximal colon, we suspected MSI-H CRC (Fig. 3a, b). Immunohistochemical examination of MMRs showed a lack of MLH1 (Fig. 4a) and PMS2 (Fig. 4b) expression in tumor nuclei, in contrast to the positive staining for MSH2 (Fig. 4c) and MSH6 (Fig. 4d). The loss of PMS2 expression is known to be followed by the loss of MLH1 expression due to heterodimerization, suggesting that this case was attributed to functional abnormality of MLH1, which is required to generate high level of MSI [5]. Lynch syndrome was not suspected because of its absence in the family and past histories; therefore, we reached a diagnosis of sporadic MSI-H CRC.

The postoperative course was uneventful. The patient did not receive adjuvant chemotherapy or other anticancer treatment. Six months after surgery, a periodic colonoscopy was performed, and no abnormal findings were seen. Subsequently, there was no evidence of cancer recurrence noted after 1 year.

\section{Discussion}

To the best of our knowledge, this is the first report of SR of primary CRC that is proven to be with MSI-H. This is an important report, since it suggests a relationship between immunological features of MSI-H and the occurrence of SR of CRC.

SR of cancer is reported to occur in approximately one in 60,000 to 100,000 people [6]. Challis et al. have

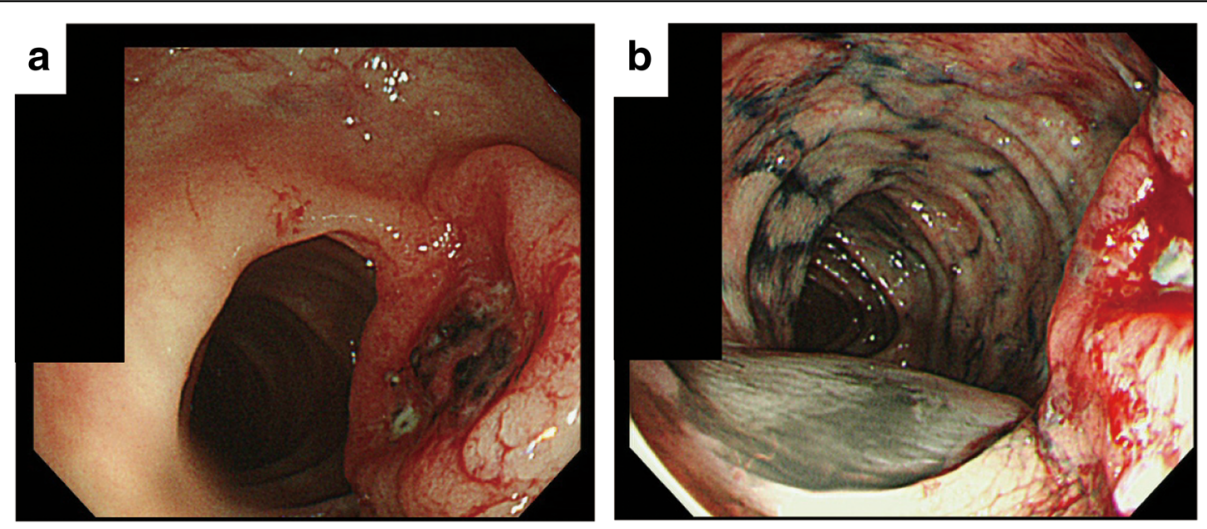

Fig. 1 a, b Colonoscopy shows a type 2 tumor in the transverse colon measuring $30 \times 30 \mathrm{~mm}$. Marking was performed near the tumor for future surgical resection 

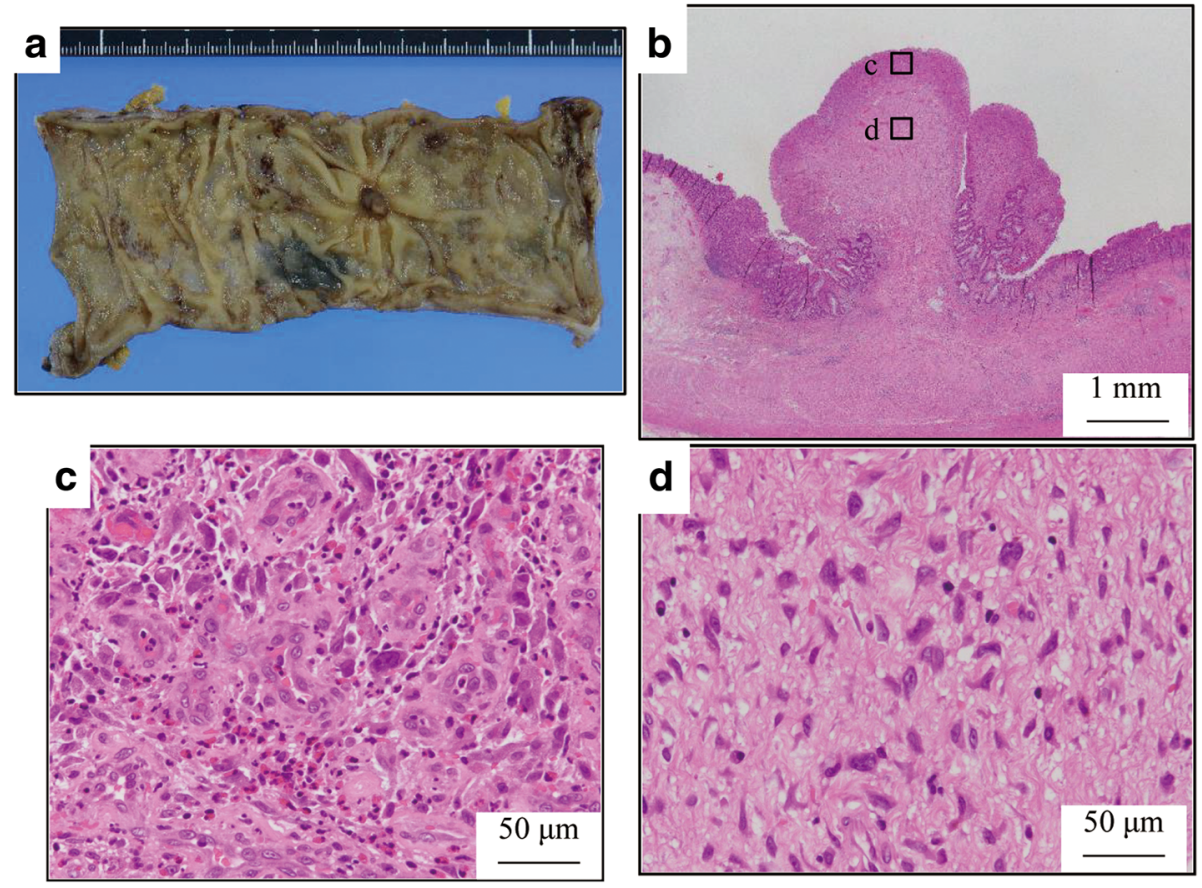

Fig. 2 The resected specimen reveals a 10-mm ulcer with plicate concentration, which has an 8.5-mm-sized polypoid lesion in the center (a). Histological examination shows marked nonspecific granulated tissue where the epithelium sloughed and fibrillization under the mucous membrane (b). No cancer cells were found in the scar tissue $(\mathbf{c}, \mathbf{d})$

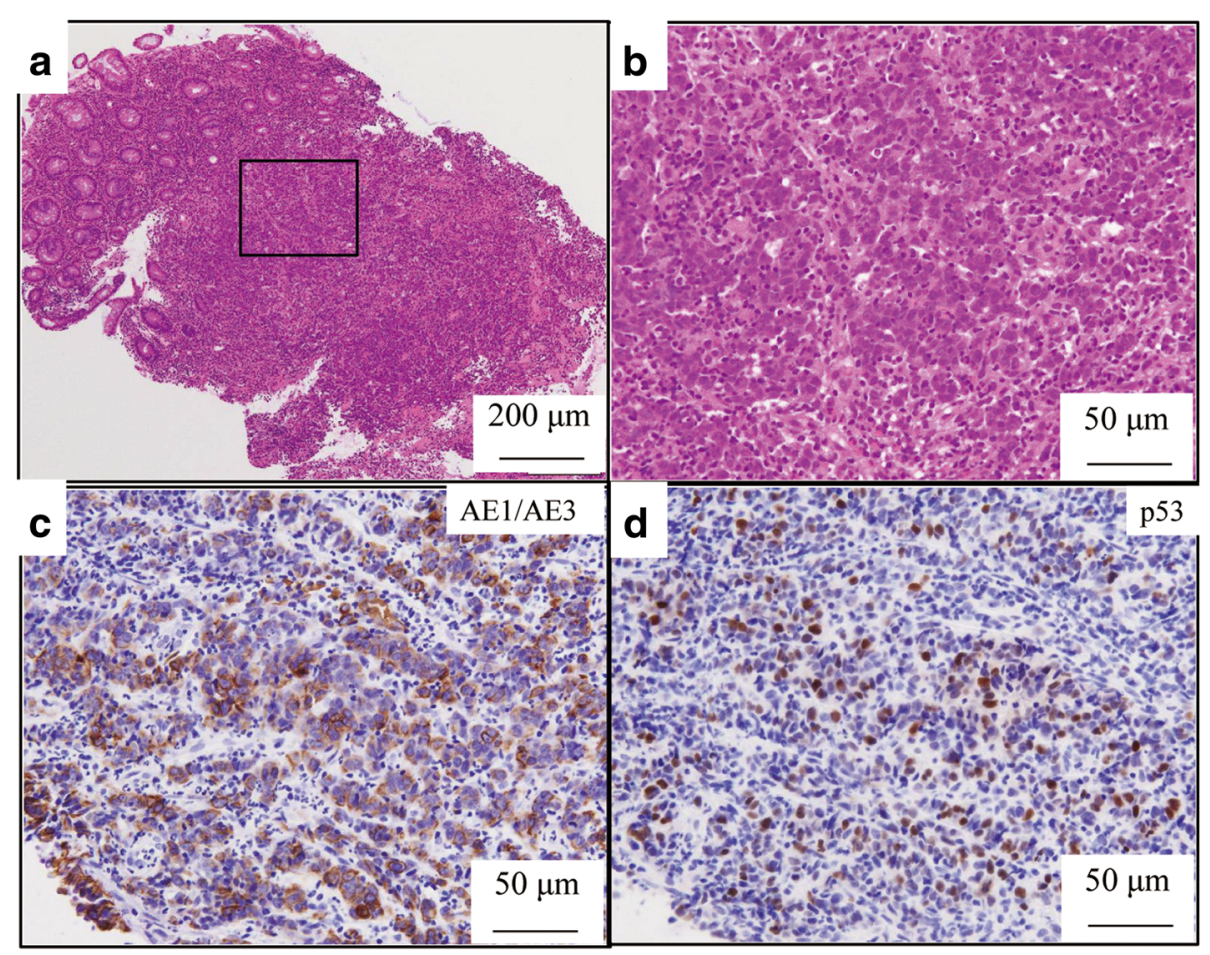

Fig. 3 Hematoxylin-eosin staining shows a poorly differentiated adenocarcinoma with tumor-infiltrating lymphocytes (TILs) in the tumor stroma $(\mathbf{a}, \mathbf{b})$. Immunohistological examination shows strong positivity for AE1/AE3 (c) and positivity for p53 (d) in the tumor; therefore, it was proven to be an adenocarcinoma 


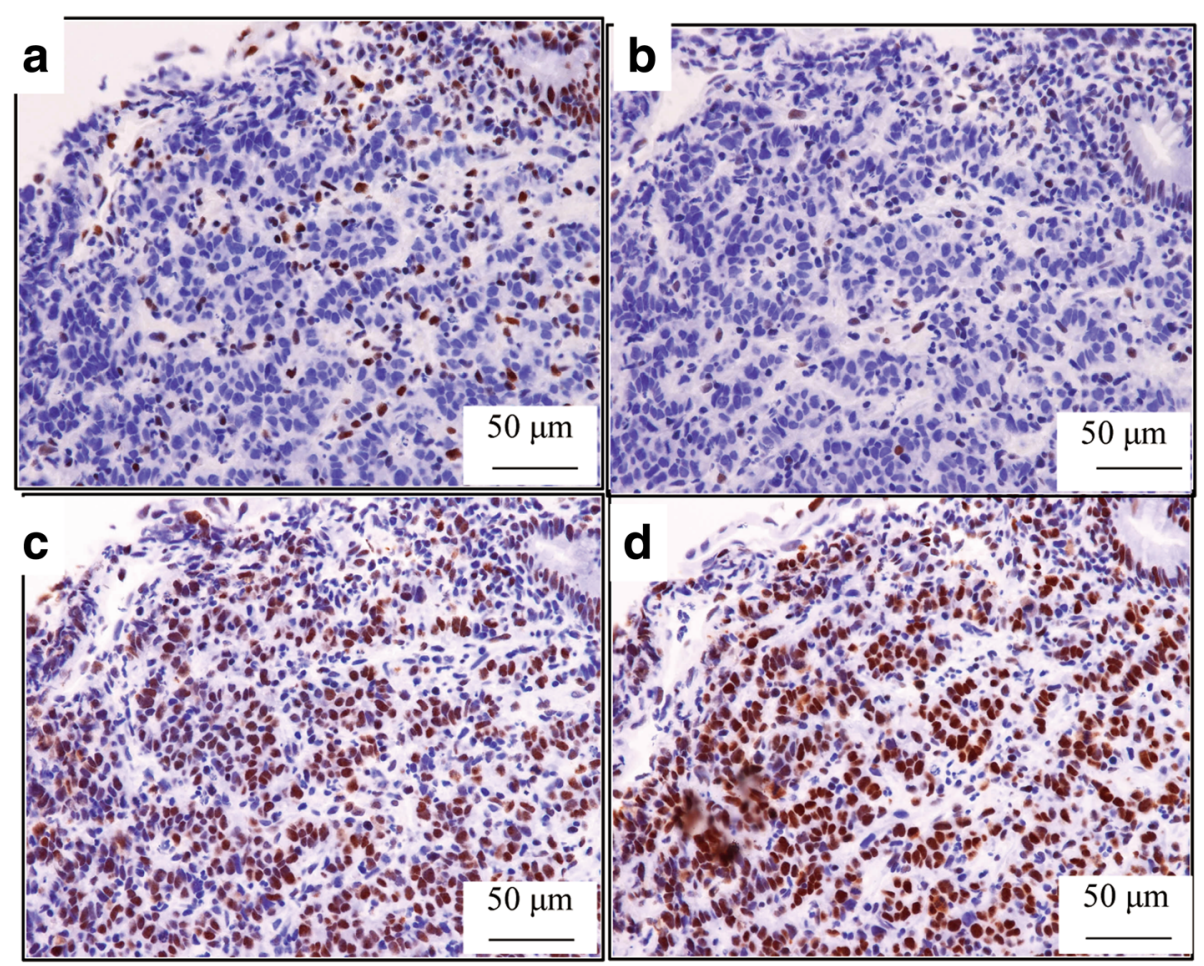

Fig. 4 Immunohistochemical examination of mismatch repair proteins shows lack of MLH1 (a) and PMS2 (b) expression in tumor nuclei, in contrast to the positive staining for MSH2 (c) and MSH6 (d). This evidence indicates that the tumor was an MSI-H CRC due to MLH1 functional abnormality. Intact nuclear staining in the stromal lymphocytes or adjacent normal epithelia was used as the internal positive control for mismatch repair proteins

reported 741 cases of SR of cancer from the year 1900 to 1987, wherein there were 99 cases of renal cancer, 92 of malignant melanoma, and 68 of malignant lymphoma. There were 18 cases of colorectal cancer, which was only $2 \%$ of all the SR cases [3]. Considering a large number of CRC cases, CRC is considered to less likely undergo SR [7].

The mechanisms of SR are still unclear. The postulated mechanisms affecting SR of cancer in general include immunological, endocrine, metabolic, surgical treatment of the primary tumor and postoperative events; elimination of a carcinogen; elimination of an antigen; angiogenesis inhibition; tumor necrosis; oncogenes; growth factors; cytokines; genetic and epigenetic; induction of benign differentiation; apoptosis; and psychological factors [8]. As for SR of CRC, the following factors were suggested: (1) ischemia by mass increase, (2) mechanical stimulation by the bowel peristaltic movement, (3) circulation imperfection by the torsion or the traction of the mass, and (4) participation of the biopsy [9]. In addition, Cole et al. have suggested that immunological reactions may cause SR by forming specific antibodies in response to the antigenic tumor cells [10]. Recently, Chida et al. have reported the possible interaction between SR of CRC and immune-mediated antitumor response, based on the presence of TILs in the biopsy specimens [11].

There are two predominant forms of genomic instability characterized by CRC: chromosome instability (CIN) and microsatellite instability (MSI). The MSI-H CRCs account for approximately $15 \%$ of all CRCs and result from dysfunction of the DNA MMR system [12]. Moreover, MSI-H CRCs show peculiar clinical-pathological features, such as prevalence in the proximal colon and histological characteristic types, which include poorly differentiated adenocarcinoma or mucinous adenocarcinoma. Although polymerase chain reaction is the standard for assessing DNA MMR competency, immunohistochemical staining for MMR proteins (e.g., MLH1, MSH2, MSH6, and PMS2) has recently emerged as a valuable complementary technique [13]. A previous study showed the loss of MMR protein expression as determined by immunohistological staining to be highly concordant with DNA-based MSI testing, with good sensitivity (>90\%) and excellent specificity (100\%) [14]. Additionally, previous clinical studies suggested that patients with MSI-H stage II or III colorectal tumors do not appear to benefit from fluoropyrimidine-based adjuvant chemotherapy [15]. MSI-H CRCs also tend to be associated with high numbers of TILs, which make them 
Table 1 Reported cases of spontaneous regression of advanced colorectal cancer (2000-2017)

\begin{tabular}{lllllllll}
\hline Author (year) & Age & Sex & Primary site & Size $(\mathrm{mm})$ & Histology & Duration (month) & Prognosis (month) & Operation \\
\hline Sakamoto (2009) [18] & 80 & M & Rectum & 25 & tub1 & 3 & ND & - \\
Shimizu (2010) [10] & 80 & M & Transverse & 25 & tub2 & 7 & (alive) & (alive) \\
Nakashima (2009) [9] & 76 & F & Ascending & 20 & tub1 & 2 & ND & + \\
Sekiguchi (2013) [15] & 69 & F & Ascending & 20 & tub2 & 1.5 & 12 (alive) & + \\
Kihara (2015) [19] & 64 & M & Transverse & 30 & tub2 & 1.5 & 12 (alive) & + \\
Chida (2017) [11] & 80 & M & Transverse & 30 & por & 1 & (alive) & + \\
Present case (2017) & 70 & M & Transverse & 30 & por & 2 & + \\
\hline
\end{tabular}

optimal candidates for immune therapy [16]. In fact, recent studies have reported the effectiveness of anti PD-1 antibody for MSI-H CRCs, because of their immunological characteristics [17].

In the case report, MSI-H was strongly suspected to be present because of the clinicopathological findings (right sided, poorly differentiated adenocarcinoma, TILs) and the MMR proteins deficiency. As for a superficial CRC with pedunculated lesions, one of the causes of SR is mechanical stimulation. However, SR due to mechanical stimulation is less likely to occur in CRC with muscular invasion, such as in the presented case. We, therefore, tried to review similar SR cases with muscular invasion to understand the mechanisms of SR and their relationships with MSI-H. A literature search was conducted using the PubMed and Ichushi databases to obtain English and Japanese literature describing SR of primary CRC with muscular invasion, and only six cases, including our case, had been reported since 2000 (Table 1). The keywords used in the search were "spontaneous regression" and "colorectal cancer." In this literature search, there were five men and two women, with a median age of 74 years, who were found to have SR of primary CRC. The median tumor size was $26 \mathrm{~mm}$, and the median duration of SR was 2.5 months. The operation was performed in six of the seven cases, and there were no cases of recurrence. In six of these seven cases, the tumor was located on the right side of the colon, and only one case was rectal in origin. Poorly differentiated adenocarcinoma was observed in two of the cases, and the other cases were of the differentiated type $[7-9,15,19]$. In this review, a tendency of the tumors to appear in the proximal colon, which is one of the features of MSI-H CRC, was observed. This finding was not conclusive because the other clinicopathological features, including histological type, were not indicative of an MSI-H phenotype. In future, further examinations are needed to determine the MSI status in other similar cases.

In our case, we do not know how or why the cancer was eliminated during the development. One of the possible hypotheses was that the cancer cells were recognized as antigens during investigations such as colonoscopy and biopsy.

\section{Conclusions}

We have presented a rare case of SR of transverse colon cancer with MSI-H. SR of CRC has a tendency to occur in the proximal colon, suggesting a possible interaction between SR of CRC and immunological features of MSI-H. Since a better understanding of this phenomenon and the mechanisms involved will have significant preventive and therapeutic implications for CRC, including anti-PD-1 immune checkpoint inhibitor therapy, further investigation is needed including testing of MSI status for such cases.

\section{Abbreviations \\ CA19-9: Carbohydrate antigen 19-9; CEA: Carcinoembryonic antigen; CIN: Chromosome instability; CRC: Colorectal cancer; CT: Computed tomography; MMRs: Major mismatch repair proteins; MSI-H: High-frequency microsatellite instability; pAf: Paroxysmal atrial fibrillation; SR: Spontaneous regression; TILs: Tumor-infiltrating lymphocytes}

\section{Acknowledgements}

We thank Dr. Daiki Taniyama and Dr. Kazuya Kuraoka (Department of Diagnostic Pathology, National Hospital Organization Kure Medical Center and Chugoku Cancer Center, Hiroshima, Japan) for their help in the interpretation of pathological data.

\section{Funding}

Not applicable.

Availability of data and materials

Data are available on request due to privacy.

\section{Authors' contributions}

MS participated in the concept/design and in drafting the article. NK contributed to the drafting and revising of the article. NK, MS, KT, SS, and TT participated in the treatment of the patient. $\mathrm{TH}, \mathrm{HE}$, and $\mathrm{HO}$ critically revised the article. TH, HY, and KM participated in the interpretation of pathological data. $T$ is the chairperson of our department and supervised in the writing of the manuscript. All authors read and approved the final manuscript.

Ethics approval and consent to participate Not applicable.

Consent for publication

Not applicable.

Competing interests

The authors declare that they have no competing interests. 


\section{Publisher's Note}

Springer Nature remains neutral with regard to jurisdictional claims in published maps and institutional affiliations.

\section{Author details}

'Department of Surgery, National Hospital Organization Higashihiroshima Medical Center, 513, Jike, Saijyo-cho, Higashihiroshima, Hiroshima 739-0041, Japan. ${ }^{2}$ Department of Surgery, National Hospital Organization Kure Medical Center and Chugoku Cancer Center, Hiroshima, Japan. ${ }^{3}$ Department of Clinical Laboratory, National Hospital Organization Kure Medical Center and Chugoku Cancer Center, Hiroshima, Japan. ${ }^{4}$ Department of Pathology, National Hospital Organization Higashihiroshima Medical Center, Hiroshima, Japan. ${ }^{5}$ Department of Gastrointestinal and Transplant Surgery, Applied Life Sciences, Institute of Biomedical and Health Sciences, Hiroshima University, Hiroshima, Japan.

\section{Received: 25 August 2018 Accepted: 23 December 2018}

Published online: 15 January 2019

\section{References}

1. Kaiser HE, Bodey B Jr, Siegel SE, Groger AM, Bodey B. Spontaneous neoplastic regression: the significance of apoptosis. In Vivo. 2000;14(6):773-88.

2. Cole WH. Spontaneous regression of cancer: the metabolic triumph of the host? Ann N Y Acad Sci. 1974;230:111-41.

3. Challis GB, Stam HJ. The spontaneous regression of cancer. A review of cases from 1900 to 1987. Acta Oncol. 1990;29(5):545-50.

4. Le DT, Uram JN, Wang H, Bartlett BR, Kemberling H, Eyring AD, et al. PD-1 blockade in tumors with mismatch-repair deficiency. N Engl J Med. 2015; 372(26):2509-20

5. Kheirelseid EA, Miller N, Chang KH, Curran C, Hennessey E, Sheehan M, et al. Mismatch repair protein expression in colorectal cancer. J Gastrointest Oncol. 2013;4(4):397-408.

6. Jerry LMCE. Textbook of family practice, 3rd edition. Saunders Philadelphia: Oncology; 1984. p. 1061-81.

7. Serizawa M, Kawarabayashi N. A case of spontaneous regression of transverse colon cancer. Gastroenterol Endosc. 2015;57(7):1490-5.

8. Abdelrazeq AS. Spontaneous regression of colorectal cancer: a review of cases from 1900 to 2005. Int J Color Dis. 2007;22(7):727-36.

9. Nakashima M, Hori K, Kimura Y, Hayashi K, Yokomizo H, et al. A case of spontaneous regression of colon cancer. The journal of the Japan Surgical Association. 2012;73(6):1482-5.

10. Shimizu H, Kochi M, Kaiga T, Mihara Y, Fujii M, et al. A case of spontaneous regression of advanced colon cancer. Anticancer Res. 2010;30(6):2351-3.

11. Chida K, Nakanishi K, Shomura H, Homma S, Hattori A, et al. Spontaneous regression of transverse colon cancer: a case report. Surg Case Rep. 2017; 3(1):65.

12. Puzzoni $M$, Silvestris $\mathrm{N}$, Leone $\mathrm{F}$, Giampieri R, Faloppi L, Demurtas $\mathrm{L}$, et al. The immune revolution in gastrointestinal tumours: leading the way or just following? Target Oncol. 2016;11(5):593-603.

13. Jung J, Kang Y, Lee YJ, Kim E, Ahn B, Lee E, et al. Comparison of the mismatch repair system between primary and metastatic colorectal cancers using immunohistochemistry. J Pathol Transl Med. 2017;51(2):129-36.

14. Lindor NM, Burgart $\mathrm{L}$, Leontovich O, Goldberg RM, Cunningham JM, Sargent DJ, et al. Immunohistochemistry versus microsatellite instability testing in phenotyping colorectal tumors. J Clin Oncol. 2002;20(4):1043-8.

15. Sekiguchi M, Mtsumoto N. Spontaneously disappearing colon cancer. Japan Gastroenterological Endoscopy Society. 2013;25:84-93.

16. Galon J, Costes A, Sanchez-Cabo F, Kirilovsky A, Mlecnik B, Lagorce-Pages C, et al. Type, density, and location of immune cells within human colorectal tumors predict clinical outcome. Science. 2006;313(5795):1960-4.

17. Passardi A, Canale M, Valgiusti M, Ulivi P. Immune checkpoints as a target for colorectal cancer treatment. Int J Mol Sci. 2017;18(6):E1324.

18. Sakamoto S, Fu K, Kobayashi O, Matsuyama S, Miyazaki A, Ogura K, et al. Spontaneous complete regression of a rectal cancer. Endoscopy. 2009;41: 910-2.

19. Kihara K, Fujita S, Ohshiro T, Yamamoto S, et al. Spontaneous regression of colon cancer. Jpn J Clin Oncol. 2015;45(1):111-4.

\section{Ready to submit your research? Choose BMC and benefit from:}

- fast, convenient online submission

- thorough peer review by experienced researchers in your field

- rapid publication on acceptance

- support for research data, including large and complex data types

- gold Open Access which fosters wider collaboration and increased citations

- maximum visibility for your research: over $100 \mathrm{M}$ website views per year

At BMC, research is always in progress.

Learn more biomedcentral.com/submissions 